\title{
Le rituel nuptial chez les Maris aujourd'hui
}

Fin des années 1990 - années 2010

\section{Viktoria Belevceva}

Traducteur : Eva Toulouze

\section{OpenEdition}

\section{Journals}

Édition électronique

URL : https://journals.openedition.org/efo/5283

DOI : $10.4000 /$ efo. 5283

ISSN : 2275-1947

Éditeur

INALCO

Édition imprimée

ISBN : 978-2-343-08571-5

ISSN : 0071-2051

\section{Référence électronique}

Viktoria Belevceva, "Le rituel nuptial chez les Maris aujourd'hui », Études finno-ougriennes [En ligne], 47 | 2015, mis en ligne le 13 juillet 2016, consulté le 20 septembre 2021. URL : http:// journals.openedition.org/efo/5283 ; DOI : https://doi.org/10.4000/efo.5283

Ce document a été généré automatiquement le 20 septembre 2021.

\section{(c) (†) 8}

Études finno-ougriennes est mis à disposition selon les termes de la Licence Creative Commons Attribution - Pas d'Utilisation Commerciale 4.0 International. 


\section{Le rituel nuptial chez les Maris aujourd'hui}

Fin des années 1990 - années 2010

\section{Viktoria Belevceva}

Traduction : Eva Toulouze

1 L'étude des rituels représente une branche à part entière de l'anthropologie : chaque peuple a son ensemble de traditions, de coutumes, de rites et de rituels, et ces derniers sont porteurs de l'expérience culturelle d'un peuple, de sa morale, de ses normes et de ses valeurs. Or si les structures rituelles d'un groupe reflètent les tendances de son évolution, elles sont néanmoins plus inertes que les transformations sociales et préservent ainsi des traits relevant des phases antérieures de cette évolution.

2 Les tendances du monde contemporain vont à l'encontre du maintien des traits ethniques des pratiques rituelles: simplification et étiolement de certaines formes, éclatement de structures naguère homogènes. Or les rituels nuptiaux se caractérisent par un haut niveau de maintien d'éléments traditionnels: il s'agit d'un élément particulièrement stable dans la culture d'un peuple. D'une part, ils permettent de préserver et de transmettre les traditions aux générations suivantes, mais d'autre part, ils peuvent être vus comme des indicateurs du niveau d'adaptation de la communauté ethnique à son environnement culturel.

La corrélation entre tradition et innovation dans les rituels familiaux et nuptiaux, l'évolution de l'institution traditionnelle de la famille et du mariage, révèlent aujourd'hui les tendances de l'évolution ethnoculturelle des Maris, leur mode d'intégration dans les processus de mondialisation et la profondeur de ceux-ci, et elles montrent l'importance du facteur ethnique dans le processus de l'identification de la personne et du groupe dans la société. Jusqu'ici, l'ethnographie marie ne s'est pas penchée spécifiquement sur cette question. Cet écrit a pour objectif de faire un catalogue de mes observations de terrain. 


\section{Rituels prénuptiaux traditionnels}

4 Certains critères permettent d'établir une typologie des formes de mariages :

5 Le type I - mariage par rapt de la fiancée, représenté par deux sous-types : le sous-type Ia, dont le rapt est l'élément rituel central, et le sous-type $\mathrm{Ib}$, où en plus du rapt, le noyau est constitué de la « petite noce » chez la fiancée, jükmaš.

6 Aujourd'hui, après le rituel proprement dit, les événements subséquents peuvent se dérouler suivant divers scénarios. Dans le premier cas, où le rapt est l'élément rituel central et unique, le mariage est aussitôt enregistré à la maison des mariages et la noce est terminée. Ce scénario peut être choisi par les jeunes pour réduire les dépenses. Plus souvent, après le rapt a lieu une fête commune et l'enregistrement du mariage est solennel. Ces deux cas de figure sont aujourd'hui relativement rares et ne se rencontrent que chez les Maris orientaux.

Chez les autres groupes de Maris, on rencontre le type II, le mariage par demande en mariage avec des variantes.

8 Bien que partout le cycle prénuptial ait été raccourci et que certains éléments rituels aient été perdus, la noce marie garde aujourd'hui des traits spécifiques, propres aux rituels traditionnels des divers groupes ethnographiques maris. Ils permettant de distinguer des ensembles rituels particuliers.

Dans le passé, un élément central des rituels nuptiaux, qui en déterminait d'autres dans le cycle nuptial, était le paiement par le fiancé de la dot, le kalym, qui la plupart du temps était lié au maintien d'une coutume archaïque, tel le rapt de la fiancée (certes, avec l'accord de celle-ci). Chez les Maris orientaux, il était répandu jusqu'au milieu du $\mathrm{XX}^{\mathrm{e}}$ siècle, mais il est en train de s'étioler de nos jours, en raison de la simplification du cycle nuptial et de la disparition du versement des dots de part et d'autre en tant que conditions sine qua non du mariage.

10 La deuxième manière de contracter le mariage - par la demande en mariage - devient la forme prédominante sur tout le territoire habité par les Maris : c'est la demande individuelle faite par le fiancé lui-même, lequel, en cas de réponse positive de la jeune fille, peut encore s'accompagner d'un envoi formel des marieurs auprès des parents de cette dernière - étape qui, néanmoins, peut être omise. En conséquence, l'accord peut se concrétiser soit directement par les jeunes gens, soit par des membres de la génération antérieure agissant en leur nom. Ce qui a changé, c'est l'ordre chronologique et la place de la demande en mariage dans la structure du rituel: par exemple, au village de Bol'šoj Ljaždur (raïon de Sernur, Mari El), la demande en mariage reste intégrée au cycle nuptial, même si elle a lieu entre un et trois jours, voire une semaine, après la cérémonie elle-même.

11 Aujourd'hui, il existe encore une manière de conclure un mariage : par l'accord mutuel des jeunes, mais sans acte rituel. Dans ce cas, néanmoins, certains éléments de la noce traditionnelle marie peuvent être inclus dans la noce proprement dite, mais en général la période prénuptiale n'est marquée par aucun rituel. C'est aujourd'hui cette forme qui est dominante dans la population marie urbaine.

Aujourd'hui, la dot a cessé d'être un élément clé du mariage, bien qu'elle n'ait pas perdu de sa signification dans les localités rurales, surtout chez les Maris orientaux des raïons d'Agryz et de Kukmor au Tatarstan, et dans les raïons de Baltačevo, de Birsk et de Bakaly au Bachkortostan, où, comme avant, une partie obligatoire de la dot se 
présente sous forme de bétail. En plus, la dot comprend des objets indispensables dans le quotidien (literie, vaisselle, affaires personnelles), éléments traditionnels de la dot des fiancées maries, ainsi que des objets liés à l'aménagement de la maison : mobilier, réfrigérateur, télévision, etc., qui souvent sont acquis conjointement par les parents des deux époux.

Dans la noce d'aujourd'hui, certains éléments du rituel prénuptial ont disparu, comme l'inspection, mais le modèle de la demande en mariage continue à fonctionner, c'est-àdire que certains éléments en sont supprimés, par exemple les cadeaux à la fiancée, könymylan solykym puymaš (litt. " présent de foulard en signe d'accord ») ou encore üdyr solyk jumo (litt. "présent de foulard à la fiancée »), l'« échange des anneaux ", šergaš vašmalyš ; l'« accord nuptial », pušlaš puaš et le "banquet de prière ». D'autres rituels, comme üdyr jümö (consommation d'alcool par la fiancée) et üdyr araka jüaš / ÿdÿrjüäš (litt. « boire l'alcool de la vierge »), d'après le témoignage d'informateurs, sont toujours en usage, mais ont perdu leur signification originelle et ne sont plus qu'un moment distrayant. D'autres ont complètement disparu, comme la conclusion de l'accord nuptial et la visite du fiancé à la fiancée pour transmettre les mesures pour les cadeaux. Quant aux rituels propres au cycle prénuptial, beaucoup d'entre eux ont cessé d'exister : l'accord du père du fiancé sur la visite à la famille pendant la cérémonie du mariage, l'inspection des biens du marié - kačynsurt-pečežym palen nalaš, la visite rituelle de l'étuve par les futurs mariés, les rituels de confection de la bière et de rangement de la dot, et enfin les traditions orales du mariage et les costumes des participants.

Un certain nombre d'autres rituels continuent d'être pratiqués, mais de manière simplifiée, comme la visite par les parents du fiancé à la maison de la fiancée, kinde pukšymaš (litt. "nourrir de pain »), kinde vaštalmaš (litt. " échange de pain »). Dans le raïon de Sernur du Marij-El, le terme désignant ce rituel a été préservé, même si dans les faits celui-ci ne recouvre plus que la collation offerte avec les nourritures apportées lors de la demande en mariage. Chez les Maris non chrétiens, dans cette même région, au moment de la demande en mariage, l'aîné du clan prononce une prière, acte qui sanctifie le contrat nuptial.

16 C'est chez les Maris orientaux que les rituels prénuptiaux se sont le mieux préservés et continuent d'exister même de nos jours : par exemple la "petite noce » chez la fiancée, jükmaš (litt. «abreuvage ») / kürojümaš (litt. «fête prénuptiale ») et le transport de la fiancée chez les parrains du fiancé, pürtomo pört.

Il faut surtout noter le rituel appelé üdyr ončyl jümö - lequel était, dans l'ordonnancement traditionnel, localisé de manière relativement étroite chez les Maris de Morko. Aujourd'hui, son espace s'est élargi, et ce rituel existe aussi bien chez les Maris des plaines que chez ceux des collines, sous forme d'un rassemblement des amies de la fiancée où l'on discute l'achat fictif de celle-ci par le fiancé et l'on met en place des scénarios possibles, des épreuves à passer et parfois le banquet.

Tout le cycle est caractérisé par la simplification des rituels dans la partie prénuptiale.

\section{Les rituels de célébration de la noce à la campagne}

19 Reste la localisation traditionnelle des éléments de la noce : chez les Maris des plaines comme chez ceux des collines, la fête principale de la noce, le lieu de la confirmation religieuse du mariage, est la demeure du fiancé. Certains rituels tels que le changement 
de la coiffe de la fiancée, qui adoptent la coiffe des femmes mariées, ou encore celui du lit, ont perdu leur signification. Chez les Maris des collines et chez une partie des Maris des plaines, la partie religieuse du mariage a lieu dans une église orthodoxe.

L'uxorilocalité de la noce est préservée chez les Maris orientaux et chez ceux de Morko. Par exemple, chez les Maris orientaux, la culmination de la noce a lieu chez les parents d'accueil du mariage: c'est là qu'ont lieu les festivités, la cérémonie religieuse, le changement de coiffe. Ce dernier, chez les Maris orientaux, a lieu effectivement; ailleurs, il existe seulement de manière symbolique, en peignant les cheveux de la mariée. Chez les Maris de Morko, les moments centraux de la noce peuvent avoir lieu aussi bien chez la mariée que chez les parents d'accueil. Chez une petite partie de ceux des Maris des plaines qui suivent les traditions "païennes », la cérémonie religieuse a lieu chez la mariée ou chez les parents d'accueil, c'est-à-dire qu'elle respecte l'uxorilocalité traditionnelle. Mais la fête aura lieu chez les parents du fiancé.

On peut ainsi conclure que malgré la simplification de certaines pratiques rituelles, les trois types de localisation des rituels nuptiaux sont préservés : virilocale, uxorilocale, et dislocale. Mais dans la noce d'aujourd'hui, il existe aussi d'autres lieux : la fête peut avoir lieu dans une cantine ou dans un café, ou encore dans une boîte de nuit ou dans une école. Dans ce dernier cas, on ne peut plus lier ce lieu à l'une des familles des nouveaux époux et le choix de l'endroit dépend de facteurs secondaires, comme le confort, la localisation, les prix, etc. Autrement, la localisation reste le village du marié ou de la mariée; ce qui change est l'endroit précis. Et si, d'après le scénario traditionnel, la fête était censée se dérouler chez les parents d'accueil, le local choisi sera situé dans le village de ces derniers.

Ce qui a aussi changé, c'est le moment où la mariée quitte la maison de ses parents : il est harmonisé avec le moment du mariage civil à la maison des mariages, où le cortège nuptial se rend directement depuis la maison de la mariée. Si la tradition prévoyait les visites, le «porte-à-porte", aujourd'hui celles-ci peuvent avoir lieu soit avant l'enregistrement solennel du mariage soit après. Dans ce dernier cas, le cortège se divise : d'une part les jeunes, avec le jeune couple et les témoins ainsi que les amis et la famille de leur génération, et d'autre part les plus âgés. Le premier groupe, avant d'arriver chez le marié, va faire une promenade en passant par les lieux mémoriaux où il se fait photographier, alors que les plus âgés, dont le groupe des femmes, süan-vate, partent «faire du porte-à-porte ». Les Maris orientaux font exception : la fête a lieu chez les parents d'accueil pürtomo pört, où la fiancée est conduite après la "petite noce » chez ses parents.

23 Ces innovations ont conduit à changer le scénario traditionnel de la noce. Par exemple, chez les Maris des collines, de nouveaux points ont été inclus dans l'itinéraire : l'arrêt obligatoire à la maison des mariages pour le mariage civil et la promenade aux lieux mémoriaux avec les photos. Mais de manière générale, l'ordonnancement du rituel traditionnel est préservé : le cortège nuptial (süan), sans visites à la famille, se rend à la maison de la mariée où il est accueilli, puis se rend directement chez le marié, ou bien part pour le mariage religieux à l'église orthodoxe.

24 Les Maris des plaines connaissent une version plus complexe du scénario nuptial: avant d'aller chez les parents de la mariée, le cortège s'arrête chez le marieur (ončyč koštšo / kokla koštšo) dans le village de la mariée; il se rendra ensuite chez les parents de la mariée ainsi que chez ceux du marié. Partout viennent s'ajouter de nouveaux éléments : le mariage civil à la maison des mariages et la promenade avec les photos. De 
plus, si la fête a lieu dans une cantine ou dans un café, le cortège nuptial va d'abord chez les parents du fiancé avant de se diriger à l'endroit où aura lieu la fête principale. On peut ajouter à ces nouveaux éléments, aussi bien chez les Maris des plaines qu'en partie chez les Maris des collines, l'arrêt du cortège nuptial devant la maternité pour déterminer le sexe du futur enfant des nouveaux mariés.

Dans les différents groupes et sous-groupes ethnographiques, des différences locales peuvent se présenter. Dans le raïon de Sernur (Mari El), le mouvement du cortège et donc le scénario lui-même sont compliqués par l'apparition d'un élément particulier : les «trois ponts ». Entre la maison de la mariée et le lieu de la fête principale, le cortège doit passer par-dessus trois ponts (ou tout au moins un pont) sur lequel le marié doit porter la mariée. Chez les Maris de Morko, le cortège doit s'arrêter chez les marieurs, aussi bien dans le village de la mariée que dans celui du marié.

La principale particularité dans le scénario de la noce chez les Maris orientaux tient à ce qu'avant la noce, une fête comparable à celle de la noce a eu lieu chez les parents de la mariée, après laquelle la mariée va vivre chez ses parents d'accueil. Ainsi, chez les Maris orientaux, on trouve deux variantes du scénario de la noce : dans la première, la mariée, à l'arrivée du cortège, se trouve chez ses parents d'accueil et dans le deuxième cas de figure, avant la rencontre avec le fiancé, elle se trouve dans « la maison de ses jeux de jeune fille", üdyr modmo pört. Ainsi, chez les Maris orientaux, le scénario traditionnel se maintient pratiquement sans modifications, à part l'inclusion des nouveaux éléments : le cortège et la famille de la mariée vont à la maison des mariages pour le mariage civil, puis une partie du groupe, avec les jeunes mariés, va faire une promenade par les lieux mémoriaux et se faire photographier; dans certains cas, le cortège part du domicile du marié pour gagner le lieu principal de la fête.

Toujours chez les Maris orientaux, certains éléments du rituel sont caractérisés par une grande variabilité. Dans les raïons de Bakaly, de Birsk et de Baltačevo, (Bachkortostan), le séjour de la fiancée chez les parents d'accueil a été substantiellement raccourci : sa durée varie entre deux semaines et trois jours; parfois, la jeune fille y est conduite seulement pour une nuit, pour ne pas enfreindre le scénario nuptial traditionnel (Babenko 1990, p. 10-11). Dans le raïon de Kukmor, au Tatarstan, on trouve trois cas de figure : 1 . La fiancée est emmenée chez les parents d'accueil pour très peu de temps ; 2 . On va la chercher dans la maison voisine, qui se présente comme une imitation de celle des parents d'accueil ; 3. Elle est " rachetée ", c'est-à-dire qu'on va la chercher chez ses parents pour partir directement à la maison des mariages.

Le temps passé par la fiancée dans la maison des parents d'accueil dépend aujourd'hui de facteurs divers et tout d'abord de la disponibilité de la fiancée elle-même, de l'existence dans la famille de personnes suffisamment proches pour être susceptibles de jouer le rôle de parents d'accueil, de leurs conditions de logement, de la distance de leur domicile, etc. Ces journées sont consacrées par la fiancée à des visites à la famille et aux voisins. Elle va en visite accompagnée de sa mère d'accueil, et laisse sur le seuil de chaque maison une pièce « de couleur blanche » (jadis, une pièce d'argent). Elle ne cesse de changer de vêtements, afin de montrer son aisance matérielle. La coutume veut qu'elle boive dans chaque maison deux tasses de thé, une pour elle et une pour son futur mari (Babenko 1990, p. 10).

29 La soirée d' »enterrement de la vie de jeune fille », üdyr modeš, a pénétré dans les coutumes contemporaines : c'est un élément obligatoire pour la plupart des noces des Maris du Bachkortostan. Jadis, c'étaient les parents d'accueil qui louaient une maison 
pour tenir cette fête, mais depuis les années 30-40 du XX $\mathrm{XX}^{\mathrm{e}}$ siècle, c'est dans la boîte de nuit du village qu'elle est organisée. Le cas échéant, on utilise une maison voisine. Dans la plupart des cas, l'üdyr modeš remplit la fonction d'un « endroit où l'on va chercher la fiancée ", sans être pour autant lié à un rituel précis. Mais dans les raïons de Mišino, de Baltačevo et dans quelques autres endroits au Bachkortostan ont été préservés quelques rituels: la triple visite au fiancé par la mère d'accueil, purtumo ava, l'organisateur de la noce sausom et le témoin kugu venge de la maison des jeux de jeune fille, les danses du kugu venge, les dialogues rituels entre les principaux participants à la noce (de nos jours, ils se présentent sous forme de plaisanteries), la couverture du visage de la mariée, le triple tour de la table fait par les principaux participants à la noce (Babenko 1990, p. 20).

Le moment même de la noce a pu changer. Chez les Maris orientaux, elle a lieu un samedi, et c'est le jeudi précédent que la jeune fille est conduite chez les parents d'accueil (ainsi la durée de son séjour dans leur maison est-elle aujourd'hui de trois jours), le mariage civil ayant lieu le vendredi (Jamurzina 2011, p. 59). Ceci a conduit à changer le moment du début du banquet nuptial : si dans la noce traditionnelle chez une partie des Maris des plaines et des Maris orientaux le rituel commençait en soirée, et la fête durait toute la nuit jusqu'à l'aube, aujourd'hui la fête commence le matin ou du moins conformément à l'heure du mariage civil.

Les éléments les plus stables dans le rituel nuptial sont la structure générale, les scénarios de déroulement, la terminologie des moments-clé et les activités correspondantes. Par exemple, le rituel du changement de coiffe a longtemps été un des moments clés du rituel nuptial. Il est étroitement lié aux représentations magiques et sociales, et il témoignait de l'association de la jeune femme à son nouveau clan. De manières différentes, il était suivi par tous les groupes ethnographiques maris. L'une des obligations de la mère d'accueil, appelée pürtymo ava, kijamat ava, svaha vate, vuj pütyryšo ava (litt. «femme enveloppant la tête »), était de retirer la coiffe de jeune fille pour la remplacer par la coiffe de femme mariée; elle devait aussi mener tous les rituels centraux avec les jeunes. Aujourd'hui, ce qui reste de ce rituel est le peignage symbolique des cheveux de la mariée, qui témoigne de son passage au statut de femme mariée.

Dans le passé, la noce traditionnelle était caractérisée par la présence de fonctions électives; aujourd'hui, on note une tendance à garder la terminologie tout en en perdant le sens profond. Ainsi, chez les Maris des plaines (raïons de Medvedevo, d'Oršanka et de Sovetskij du Mari El) les ončal šogošo üdyr et kugu venge ont assumé la fonction des témoins homme et femme au mariage civil (dans certains cas aussi au mariage religieux), alors que l'ončyč šogošo üdyr aide la mariée à organiser sa vente fictive, et que le kugu venge aide au déroulement de celle-ci ; mais avec la simplification des rituels traditionnels, les fonctions traditionnelles vont-elles aussi en se perdant.

Chez les Maris non baptisés, l'acte traditionnel de confirmation du mariage impliquait un rituel animé par le kart (le prêtre de la religion traditionnelle marie), mais aujourd'hui cette figure a changé et c'est l'ordonnateur de toute la cérémonie qui officie. Chez les Maris orientaux, c'est une paire de femmes, les kart vate, qui jouent ce rôle et assurent la composante d'oralité du rituel. Les rôles clé du rituel ont également subi des modifications : l'ončal šogošo üdyr et le kugu venge chez les Maris orientaux étaient jadis les substituts du marié et de la mariée dans certaines activités rituelles; de nos jours, ils ont perdu une partie de leurs fonctions, et ne sont plus que les témoins du 
mariage civil. Comme avant, les süan vate (litt. « femmes du cortège ») bénéficient d'un statut élevé : avec les musiciens, elles assurent les chants pendant tout le cycle. Le saus/ sabus, pour sa part, a maintenu l'essentiel de sa fonction : comme avant, il ordonne l'ensemble des festivités. Mais chez les Maris orientaux, certaines de ses fonctions sont passées au kart, et chez les Maris des plaines au kugu venge, ce qui a eu comme effet de réduire quelque peu l'importance du saus dans le rituel.

Dans la noce à Morko, comme c'était le cas avant, un rôle central est dévolu à la mère d'accueil, qui intervient dans pratiquement tous les rituels du cycle nuptial. Il faut noter que ce personnage n'y a pas gardé seulement sa fonction, mais aussi son costume.

Aujourd'hui, les cérémonies religieuses, aussi bien païennes qu'orthodoxes restent un point culminant de la noce traditionnelle. Mais la dimension religieuse du mariage a cessé d'être obligatoire et reste au choix des mariés. Là aussi, nous pouvons identifier des tendances générales. Les Maris des collines se marient plus que les autres religieusement. Les Maris des plaines peuvent eux aussi le faire pendant la noce, mais en général ils ont tendance à reporter cette cérémonie à une période plus tardive, une fois la noce passée. Les Maris des plaines non baptisés pratiquent le rituel païen du mariage aussi bien dans la maison de la mariée que dans celle du marié. Mais la fonction d'officiant revient soit au père du marié, kače ača, soit au süan vuj, le responsable du cortège.

Chez les Maris des plaines des raïons de Sernur, de Novyj Tor"jal et de Paran'ga dans la République de Mari El, où Maris baptisés et Maris non baptisés coexistent, le rituel païen n'est pas perçu par les invités et par les mariés comme un rituel religieux, bien qu'il se compose d'importantes activités rituelles: on allume des bougies, on dit des prières, on partage des aliments rituels, on prépare une table sacrificielle couverte d'assiettes avec des mets rituels, avant que son contenu soit distribué aux enfants, ou encore réservé au chien. La prière peut contenir soit une adresse à Kugu Jumo, soit un texte de prière orthodoxe, voire, la plupart du temps, les deux. Chez les Maris de Sernur, ce rituel peut voir l'intervention de diverses personnes. La prière peut être dite aussi bien par le doyen du clan, le töryštö, que par tout parent âgé du marié ou de la mariée. C'est le père du marié, le kače ača, qui a le droit de distribuer la nourriture rituelle, mais en cas de besoin il peut être remplacé par le père d'accueil. La composition des officiants peut varier considérablement même dans un seul et unique raïon, suivant la maîtrise de la tradition dans les différentes familles. Aujourd'hui, les personnes ayant la position la plus active dans le suivi du scénario traditionnel sont les femmes âgées, qui peuvent intervenir aussi bien pour accompagner l'activité rituelle qu'en officiant elles-mêmes.

L'un des éléments les plus stables et les plus caractéristiques de la noce traditionnelle marie est la distribution de cadeaux de la part de la mariée, aussi bien à sa famille qu'à celle de son fiancé. On peut suivre l'évolution de ce rituel dans le changement des matériaux utilisés pour confectionner les cadeaux. Comme avant, ceux-ci sont des serviettes, des chemises et des robes, mais ils sont confectionnés en tissus industriels. Les vêtements de coupe traditionnelle et les décors sont commandés à des artisans, ou bien sont réalisés avec le concours de parents ou de voisins. La mariée prête une attention toute particulière aux cadeaux destinés à la famille de son futur mari et à ses parents d'accueil.

D'importantes transformations ont également eu lieu dans le domaine de la cuisine nuptiale. On y voit aujourd'hui des plats de la cuisine internationale, qui se sont 
répandus partout au $\mathrm{XX}^{\mathrm{e}}$ siècle: des salades, des tartes, etc. On offre aux invités presque exclusivement de l'alcool industriel. Mais un certain nombre de mets traditionnels demeurent de rigueur à des moments précis de la cérémonie nuptiale, car ils ont une valeur sacrale. Par exemple, dans le village de Narat-Čukur (raïon de Bakaly, République du Bachkortostan), l'accueil dans la famille du marié comprend trois mets : des melna (crêpes), des peremeč (gâteaux au fromage blanc avec des graines de chanvre, de la purée de pommes de terre ou des baies), et des tuara (galettes au fromage blanc). On trouve encore aujourd'hui chez les Maris orientaux toute une série de noms spécifiques pour les plats de cérémonie, qui ailleurs sont appelés autrement - laška, sokta, tuvyrtyš, kogyl'o, komanmelna, etc. (Sepeev 1967, p. 131). Certes, la quantité de plats proposés a diminué par rapport aux menus traditionnels, qui comportaient entre sept et douze mets.

L'ancien costume nuptial traditionnel n'est plus utilisé aujourd'hui. Les mariés préfèrent un habillement contemporain (robe blanche et costume masculin classique); mais les femmes du cortège, appelées süan-vate, gardent le costume traditionnel et ce type de vêtements reste un cadeau prestigieux à la famille.

En revanche, les rituels magiques destinés à protéger le jeune couple se distinguent par leur stabilité. Les matières premières archaïques (feutre, houblon) n'y ont pas perdu de leur importance. Par exemple, quand le défilé était accueilli dans la maison du marié, les mariés étaient obligatoirement installés à table sur des sièges couverts de feutre. Avant de quitter le domicile de la fiancée ou des parents d'accueil, les principaux participants à la noce faisaient trois fois le tour de la table, ce qui symbolisait l'adieu aux esprits de la maison. Dans certains raïons habités par les Maris, ces rituels subsistent. Par exemple au village de Mari-Kupta, dans le raïon de Marij-Turek (Mari El), avant le départ de la mariée du domicile de ses parents, le fiancé met de l'argent dans le sein de la mère de sa future femme (aujourd'hui, il fait semblant de le lui mettre dans la main), ce qui représente le rituel cyzy pukšy-mylan (litt. « pour l'alimentation au sein »). L'un des éléments culminants de la noce traditionnelle - l'échange de la coiffe de jeune fille pour la coiffe de la femme mariée - est représenté chez les Maris orientaux par une pratique fortement modifiée: non seulement les cheveux de la mariée sont peignés, mais le foulard est retiré de la tête de la nouvelle épouse.

La pratique de se rendre à une source, extrêmement significative dans le rituel nuptial chez pratiquement tous les peuples de l'Oural et de la Volga, se perd peu à peu, compte de tenu de la perte de son importance utilitaire. Mais les personnes âgées et celles d'âge moyen considèrent ce moment comme une partie incontournable du cycle nuptial et en soulignent l'importance sacrale. Quelques phénomènes résiduels peuvent être interprétés comme des évolutions de ce rituel : par exemple, dans le raïon d'Oršanka, ce rituel a été sorti du cycle nuptial ; mais la première fois que la jeune mariée se rend à la source, elle jette une pièce de monnaie dans l'eau. Dans certains cas, ce rituel acquiert une signification nouvelle et devient une composante ludique de l'activité nuptiale comme c'est le cas par exemple au village de Bol'šoj Serdež dans le raïon de Sernur (Mari El) : la jeune fille va chercher de l'eau, et les jeunes du village du marié (garçons et filles) essaient de l'en empêcher ; si elle n'arrive pas à ramener toute l'eau, elle doit se racheter. Au village de Čibyž (raïon de Sernur, Mari El), les témoins participent activement à ce rituel : la femme témoin offre à son homologue masculin une serviette, pour que celui-ci aide la mariée à rapporter l'eau sans empêchements. 

qui permettaient de vérifier les savoir-faire (préparation de crêpes, nettoyage du plancher, etc.) de la jeune mariée le lendemain du mariage, rituels qui se présentaient de manière différente suivant les groupes. Par exemple, dans le raïon de Sernur, la jeune fille doit faire des crêpes qui seront ensuite offertes à la famille. Au village de Počinok-Kučuk (raïon de Kukmor, République du Tatarstan), elle offre de l'alcool aux invités, qui répondent en jetant par terre de l'argent. Au village de Nurma (raïon de Medvedevo, Mari El), la belle-mère, pour soulager sa belle-fille de ses tâches, l'aide ou bien reprend entièrement son travail à son compte. Dans les cas où la deuxième journée se déroule dans la nature, par exemple au village de Marij Kupta dans le raïon de MarijTurek (Mari El), c'est là qu'a lieu l'imitation de ce rituel : la jeune mariée doit accomplir des tâches liées soit à la cuisine soit au ménage. Les crêpes faites par la jeune mariée, or'en melna, le deuxième jour, sont le plat principal offert aux participants dans les noces des Maris orientaux des raïons de Birsk, de Bakaly et de Baltačevo (Bachkortostan). Si la jeune mariée ne sait pas ou ne veut pas faire les crêpes, elle emploie une femme qui la remplace et à laquelle elle offre une serviette ou de l'argent. Encore aujourd'hui, chez les Maris orientaux, et chez une partie des Maris des plaines, on croit que si les crêpes sont brûlées, la mariée ne sera pas heureuse avec son époux (Babenko 1990, p. 25). Dans le raïon de Kaltasy (Bachkortostan), le matin du deuxième jour de la noce, la jeune mariée chauffe le sauna et y laisse une petite rançon, dont devra s'emparer quelqu'un de la famille de son mari (Babenko 1990, p. 26). Mais il s'agit là d'un rituel étroitement localisé; par exemple dans le raïon de Mišino (Bachkortostan), où la noce marie a gardé beaucoup de traits traditionnels, ce rituel n'existe pas. On peut en déduire que cet élément est un emprunt tardif, sans doute aux traditions russes.

On peut également identifier dans le cycle nuptial un rituel qui a repris de l'actualité : la jeune épouse doit changer l'intérieur du domicile du marié, suspendre de nouveaux rideaux, mettre une nappe sur la table, des tapisseries, etc. Mais il est peut-être seulement une modification d'un rituel qui a existé dans le passé, celui de la « décoration de la maison» du marié par la mariée, qui faisait appel aux éléments de son trousseau (serviettes, etc.) (Babenko 1990, p. 26).

\section{Les rituels postnuptiaux}

Le cycle des rituels postnuptiaux a également considérablement évolué : réduction des visites des nouveaux mariés à leurs familles, nombre des collations et nombre des invités, réduction des cadeaux des deux côtés. Dans certaines localités, cependant, il reste quelque chose des anciennes traditions : par exemple dans le raïons d'Oršanka (Mari El), l'un des premiers rituels postnuptiaux, destiné à intégrer la jeune mariée au clan de son mari, le premier sauna, est l'occasion pour la jeune femme de faire de nouveaux cadeaux à sa belle-mère, lesquels, d'après le témoignage d'informateurs, sont encore plus chers que ceux faits lors de la noce. La nouvelle épouse avait comme obligation d'habiller sa belle-mère à neuf, avec la coiffe et les sous-vêtements. Aujourd'hui, ces cadeaux se composent de sous-vêtements et de vêtements achetés, de coupes de tissu; les robes traditionnelles maries, confectionnées à la demande, restent un cadeau prestigieux.

Études finno-ougriennes, 47 | 2015 
D'autres rituels ont pratiquement perdu leur signification: il s'agit de tous ceux destinés à protéger les époux ainsi que ceux au cours desquels ces derniers évitaient les parents les plus âgés; cela ne pouvait s'arrêter qu'avec une levée rituelle des interdits. Chez les Maris, comme chez les autres peuples du cours moyen de la Volga, ces rituels postnuptiaux avaient comme objectif de renforcer l'entrée de la jeune femme dans le clan de son mari et de l'intégrer parmi les femmes mariées de celui-ci; ils servaient aussi à renforcer les liens entre son clan d'origine et celui de son mari.

En conclusion, des changements qualitatifs considérables dans le rituel nuptial mari traditionnel ont conduit à la formation d'un type de rituel où pratiques contemporaines et pratiques traditionnelles coexistent, et où la variation est considérable suivant les niveaux d'intégration de ces différents éléments. L'apparition de nouvelles étapes dans le rituel nuptial est due à plusieurs facteurs, surtout d'ordre sociopolitique: la propagande de nouveaux rituels soviétiques, la multiplication des mariages mixtes, la mobilité de la population et les processus d'urbanisation.

Néanmoins, un certain nombre de points culminants de ces rituels ont subsisté grâce à la persistance des relations entre clans, à l'isolement relatif du milieu rural, qui reste le milieu dominant pour la majorité de la population marie, au maintien des conceptions religieuses traditionnelles. Indiscutablement, la noce traditionnelle marie est organique, son caractère original se manifeste dans les chants, dans les danses, dans les mets rituels, dans les symboles traditionnels, et dans de nombreuses activités. Aujourd'hui, le déroulement de la cérémonie nuptiale dépend de nombreux facteurs, depuis la localité où aura lieu la cérémonie jusqu'aux préférences personnelles des jeunes mariés; il dépend aussi de la connaissance des traditions par les membres de leurs familles. On peut parler aujourd'hui d'un processus inaccompli de formation d'un type de rituel nuptial contemporain.

La difficulté à préserver les formes traditionnelles tient entre autres aux processus d'urbanisation. En ville aujourd'hui, il n'est pas possible de suivre pleinement nombre d'éléments traditionnels des rituels nuptiaux. Cela tient aussi au déclin du rôle des groupes de parenté dans la préparation et dans le déroulement de la noce.

\section{Les rituels nuptiaux des maris en ville}

49 Je me concentrerai ici d'une part sur le milieu urbain de la grande ville, avec ses tendances à la standardisation de la culture, et d'autre part sur l'espace de la petite ville, où les processus d'urbanisation sont légèrement distincts. L'émergence de types divers de noces dans différents milieux culturels permet de relever les éléments stables de ce phénomène, leur maintien ou la substitution de leur destination fonctionnelle, le rôle des contacts interethniques. Cette étude permet de réfléchir à l'état des valeurs familiales et conjugales, aux stéréotypes existant dans la société, de suivre les mutations dans les fonctions de la famille ainsi que les mécanismes de fonctionnement et de transposition de la tradition.

Depuis le milieu du XXe siècle, on voit se mettre en place la noce urbaine en tant que type spécifique de rituel nuptial. Parmi les Maris, ce type de noce n'était pas très répandu jusqu'aux années 1970, même si peu à peu, ceux qui résidaient dans de grandes villes telles que Joškar Ola, Ufa ou Kazan', commençaient à assimiler la culture urbaine et à en adopter quelques traits, par exemple justement dans le rituel nuptial. Or il est 
particulièrement important de suivre ces processus, car la population marie urbaine est en augmentation régulière jusqu'à aujourd'hui.

Dans les villes moyennes, comme Volžsk ou Koz'modem'jansk, on voit se former une variante sui generis de la noce urbaine, dans laquelle on trouve à égalité des éléments standardisés de la noce urbaine (mariage civil à la maison des mariages, visite des monuments avec dépôt de gerbe, photos) et quelques éléments de la noce traditionnelle. C'est le cas, dans le cycle prénuptial, de la demande en mariage; il est vrai que celle-ci a subi des transformations fonctionnelles, mais elle n'en a pas moins préservé certains traits traditionnels. Elle permet souvent par exemple aux parents du fiancé et à ceux de la fiancée de se connaître, ce qui dans ce cas n'a pas une aussi grande importance du point de vue de la décision finale que dans la noce traditionnelle, puisque la décision a déjà été prise par les parties directement concernées. Il faut noter d'ailleurs que c'est lors de la demande en mariage que se discutent des questions traditionnelles telles que la contribution du marié (la plupart du temps, cela touche à la quantité d'alcool à offrir lors des noces) et la dot de la mariée, ainsi que la quantité et la valeur marchande des cadeaux à offrir à la famille du marié. Résumons : si jadis toutes les dépenses pour les cadeaux destinés à la famille du marié reposaient sur la famille de la jeune fille, aujourd'hui le coût en est partagé entre les deux parties. Certaines formes subsistent sous leur aspect ludique, comme la pratique de faire boire à la fiancée de l'alcool, üdyr jümo, en signe d'accord. Il reste également la distribution de cadeaux au moment de la demande en mariage, et quelques éléments de cuisine traditionnelle : par exemple, les parents, en attendant les demandeurs en mariage, mettent sur la table des plats traditionnels maris, les peremeč, les melna, les tuara. À leur tour, les membres de la famille du fiancé arriveront avec des cadeaux équivalents. À Koz'modem'jansk, dans le cycle prénuptial, la pratique du šergaš vašmalyš demeure, mais ce sont les futurs mariés eux-mêmes qui l'accomplissent. C'est l'équivalent des fiançailles et en général le fiancé offre à la jeune fille un anneau, signe de son intention de l'épouser.

Parmi les éléments caractéristiques de la noce urbaine, il faut mentionner les soirées d'adieu à la vie de garçon et de jeune fille la veille du mariage. On accorde également une grande attention à l'achat de la robe de mariée; parfois, conformément à la tradition, des informateurs nous ont dit que c'est au fiancé qu'il revient de fournir à la jeune fille la parure nuptiale.

53 Le jour convenu, le cortège nuptial du garçon (aujourd'hui, il s'agit d'une file de voitures décorées) va chercher la fiancée. Dans les petites villes, il existe toujours dans le cortège les fonctions de suan dans le cortège du fiancé et de počeš tolšo dans le cortège de la fiancée, mais en règle générale ce sont là des titres qui ne s'accompagnent pas de tâches particulières dans le déroulement de la noce. Par exemple, la mère d'accueil, qui dans la noce traditionnelle dirigeait le rituel de l'échange de la coiffe, aide aujourd'hui la mariée à distribuer les cadeaux à la famille.

Dans les petites villes de la République du Marij-El il existe de nos jours deux versions d'une noce «traditionnelle»: dans le premier cas, les participants se présentent en vêtement de ville; dans le deuxième, on garde le cortège de femmes, le suan vate: dans ce cas, les chants nuptiaux sont chantés en costume traditionnel. Le scénario de la noce est simplifié : le cortège va directement chercher la fiancée. Ensuite vient le moment de son rachat burlesque par le fiancé : le garçon est soumis à quelques épreuves peu signifiantes pour racheter la mariée aux amies de celle-ci. Après cet épisode et une petite collation dans l'appartement ou dans la maison de la mariée, les participants à la 
noce, y compris les parents de la jeune fille, se dirigent vers la maison des mariages pour le mariage civil. En cas de présence des suan vate, celles-ci chantent des chants nuptiaux également devant la maison des mariages. Après le mariage civil a lieu la promenade avec les photos, après quoi la procession nuptiale se rend soit chez le fiancé, soit dans un local de restauration pour le banquet. Il faut noter que le rôle des parents du fiancé est susceptible de variations: dans le premier modèle, après le mariage civil ils quittent le cortège nuptial et rentrent chez eux, pour y accueillir ensuite les jeunes mariés avec le "pain et le sel »; dans le deuxième cas également, ils quittent le reste des invités après le mariage civil et ne participent pas à la promenade et aux photos; ils se rendent directement au café ou à la cantine où aura lieu la fête, et c'est là qu'ils accueillent les nouveaux mariés avec «le pain et le sel». Si les invités poursuivent les festivités dans la maison du marié, les parents de ce dernier quittent également la maison des mariages avant les autres et accueillent les nouveaux mariés comme dans les autres cas.

L'accueil de la mariée dans la maison du marié était l'un des points culminants de la noce traditionnelle; on ne préserve plus de nos jours que quelques éléments de ce rituel, qui ont pour objectif de protéger les jeunes mariés des forces malveillantes. On fait appel aux mets traditionnels - les tuara, les peremeč, le beurre. Il est curieux de remarquer qu'en général, si cet accueil a lieu à la maison, on prépare les mets traditionnels (les melna, les tuara), alors que s'il a lieu dans un café ou dans une cantine, on fera appel à un pain russe standard avec du sel.

Quand la fête a lieu dans un local et que la procession est suivie par les suan vate, tout le monde se change au début de la fête et revêt l'habillement urbain ordinaire. L'une des tendances les plus récentes dans les noces maries en ville est le recrutement d'un tamada mari, et dans ce cas la noce aura lieu en mari avec des chants maris.

Lors du banquet, qu'il ait lieu dans une cantine ou à la maison, la mariée fait des cadeaux à la famille de son mari et aux invités. Ceux-ci, à leur tour, font également des cadeaux au jeune couple, surtout de l'argent. Après le banquet, les invités se dispersent. Les cérémonies du lendemain et les rituels postnuptiaux ont pratiquement disparu; il est rare que soient préservés uniquement le rituel de la confection des crêpes par la jeune mariée et les visites rendues à la famille dans les semaines qui suivent. Le deuxième jour de la noce est en général une fête des jeunes dans des cafés ou dans la nature.

Voyons maintenant un autre sous-type, la noce dans les villes grandes et moyennes, sur la base de l'exemple de Joškar-Ola. S'y trouve préservée la pratique symbolique de la demande en mariage, les noms des personnes chargées de différentes tâches pendant la noce, comme saus, ončal šogošo üdyr; mais seuls les titres ont été préservés, les tâches elles-mêmes sont prises en charge par les témoins des deux sexes. Le scénario lui-même se présente comme suit : le marié va chercher la mariée, le « rachat » a lieu suivant un scénario standardisé et s'achève avec une collation chez la mariée et le départ de tous les invités, y compris les parents de la mariée, pour la maison des mariages. Ensuite, le cortège part pour la promenade, pour les photos, et ensuite le banquet a lieu dans un café ou une cantine, ou encore chez les mariés. C'est seulement dans des cas rares, voire uniques, que des suan vate participent à la noce; l'accueil des nouveaux mariés par les parents du marié existe toujours, mais il a été modifié avec la reprise de la tradition russe $d u$ "pain et du sel» (au lieu des crêpes traditionnelles maries, les melna, du gâteau au fromage blanc tuara et du beurre). 
59 La dimension ethnique se manifeste dans les initiatives de l'animateur mari invité et dans le rituel de la distribution des cadeaux - chemises, serviettes ou robes. Ces derniers temps, on note une tendance à offrir à la belle-mère, lors de noces riches, une robe moderne avec des motifs de broderie marie traditionnelle. Une autre tendance à la mode ces dernières années est d'inclure dans l'itinéraire de la promenade, après la maison des mariages, un passage par la maternité, pour jouer à deviner le sexe de l'enfant à venir. On visite également les lieux suivants : l' » Obélisque de la gloire » et la «Flamme éternelle», le monument à la jeune famille, le «banc de la fidélité et de l'amour ", ainsi que le monument à saint Pierre et sainte Fevronia. L'une des tendances de la noce civile à la mode est d'accrocher des verrous avec les noms des jeunes mariés au pont sur la Kokšaga ; la clé du verrou est jetée à l'eau et symbolise l'indissolubilité de l'union du couple. Dans les vingt dernières années, on célèbre de plus en plus souvent le mariage religieux le jour du mariage civil.

60 Tout autre est la stratégie de comportement des Maris qui partent s'installer pour longtemps dans les grandes villes de Russie (Moscou, Saint-Pétersbourg, Ekaterinburg, etc.) et du monde. Les traditions nuptiales de leur peuple perdent pour eux de leur importance, elles y sont rejetées et la noce, en règle générale, suit la cérémonie civile ordinaire.

61 Ainsi, le rituel nuptial urbain n'est pas un phénomène homogène, on peut en distinguer plusieurs sous-types suivant un certain nombre de critères.

62 Le sous-type I inclut l'ensemble des trois cycles nuptiaux. Il est caractéristique des villes moyennes, des chefs-lieux, tels que Volžsk, Koz'modem'jansk, Zvenigovo, etc. De par sa structure, c'est lui qui se rapproche le plus du type traditionnel: dès le cycle prénuptial, il inclut la demande en mariage üdyrjüktymašt / ÿdÿrjü̈̈š, qui souvent est purement nominale, la décision du mariage ayant été prise depuis longtemps. La cérémonie centrale du mariage se présente avec des variations : elle peut avoir lieu au domicile des parents du marié ou encore dans un local de restauration, bien que cette deuxième version ait tendance à se diffuser. L'itinéraire du cortège nuptial a changé conformément aux demandes de l'époque: outre les arrêts traditionnels, il inclut la maison des mariages et la promenade avec les séances photo des jeunes mariés. Le mariage religieux n'est pas aujourd'hui un élément obligatoire du rituel nuptial, c'est pourquoi sa présence ou non-relève exclusivement du désir des nouveaux mariés et de leurs familles. Dans les petites villes, la composition du cortège nuptial se présente sous une forme réduite : le cortège du marié, süan, comprend le responsable de la noce, le süanvuj, le substitut du marié kugu venge, les parents d'accueil, les süan vate et des musiciens; dans le cortège de la mariée, počeš tolšo, les fonctions les plus actives sont celles des parents d'accueil et de la meilleure amie de la mariée. Les variations peuvent être considérables en matière de costume nuptial et de folklore. Ces éléments dépendent avant tout de la présence, dans la noce, des süan vate. Si elles sont là, le costume et les chants traditionnels dépendent entièrement de l'endroit où a lieu la noce, et correspondent aux traditions locales.

63 En ville, certains éléments stables du rituel continuent à exister, comme la collation offerte aux familles et aux invités, la bénédiction de l'icône avant le départ du cortège nuptial süan pour aller chercher la mariée, les cadeaux lors de l'arrivée du cortège chez la fiancée, les épreuves ludiques auxquelles est soumis le fiancé. Les rituels lors du départ du cortège depuis la maison des parents de la fiancée se sont conservés sans transformations importantes. Comme avant, les parents le bénissent avant son départ, 
il fait trois fois le tour de la table nuptiale, la famille de la mariée reçoit des cadeaux, on rachète la dot, on effectue le "paiement pour la nourriture au sein ». Lors de l'accueil par les parents de la mariée (que ce soit à domicile ou dans un local de restauration), les rituels suivants ont toujours lieu : réception des mets rituels, distribution de cadeaux aux parents et à la famille du marié, cérémonie sans laquelle il n'y a pas de noce marie. Le cycle postnuptial peut inclure la confection des crêpes le deuxième jour de la noce avec les cadeaux. Quant aux visites postnuptiales, elles ont perdu leur dimension rituelle et ne sont pas marquées chronologiquement.

Dans les très grandes villes, on note une réduction sérieuse des éléments traditionnels de la noce. Là, le rôle central revient aux mariés, qui déterminent le scénario de leur noce. Si les mariés choisissent une noce urbaine avec les éléments traditionnels, elle sera précédée de la demande en mariage üdyrjüktymašt / ÿdÿrjüäš. On accorde une importance particulière à la cérémonie nuptiale, qui inclut l'adieu à la vie de jeune fille / de garçon, une expédition commune d'achats de cadeaux aux parents de la mariée et du marié, la remise de l'invitation à la noce. L'événement central a lieu dans un café ou dans une cantine. Même dans une très grande ville, les Maris ont conservé quelques pratiques rituelles : la bénédiction par les parents de la mariée avant le départ du cortège nuptial, l'accueil de la mariée par les parents du marié, les mets rituels, les cadeaux aux parents et à la famille du marié. En ville, les éléments du cycle postnuptial ont pratiquement disparu, avec comme rare exception la confection de crêpes par la mariée le lendemain de la noce.

Chez les Maris orientaux, le scénario de la noce urbaine est très semblable, mais quelques traits caractéristiques le distinguent de la version de base. La différence principale est le maintien de quelques traditions archaïques et une plus grande similitude avec le scénario traditionnel de la noce. Une "petite noce » a lieu dans la maison de la fiancée, qui inclut en partie la demande en mariage. De plus, suivant un scénario traditionnel, le marié va en règle générale chercher la mariée non pas chez les parents de celle-ci, mais chez les parents d'accueil, kiamat ača et ava ou encore à la «maison des jeux de jeune fille ", üdyrmodmo pört, qui peut être l'appartement de tel ou tel membre de sa famille ou de voisins. La dimension religieuse de la noce est obligatoire et se concrétise par une prière.

De nos jours, dans les petites villes et dans les villes moyennes, la noce représente un phénomène très instable, dont le déroulement dépend de nombreux facteurs: la solidité des liens familiaux, la proximité territoriale du village, la conscience de l'importance de la tradition, etc. La vitalité des éléments traditionnels dépend du niveau de conviction, chez les personnes âgées ou d'âge moyen, de la nécessité de respecter certains actes rituels afin de garantir la réussite de la vie conjugale du jeune couple, qui en général les accepte volontiers. C'est pourquoi souvent, dans les très grandes villes, la noce peut suivre un scénario du premier sous-type, alors qu'au contraire, dans des petites villes, seulement certaines noces ont lieu suivant un scénario simplifié. De manière générale, les Maris vivant en ville préservent les éléments rituels principaux avec leurs spécificités régionales, même dans des conditions socioculturelles nouvelles. Cependant, les conditions de la ville (structure de l'espace, emploi) ne sont pas favorables au scénario traditionnel de la noce : celui-ci a été raccourci et les activités principales se déroulent dans des lieux publics (de restauration). Un facteur non négligeable de transformation du cérémonial traditionnel et de mise en place d'un nouvel ensemble rituel est la tendance à la standardisation de 
l'espace socioculturel de la ville sous l'influence de phénomènes tels que la mode, la " culture pop ", etc.; il ne faudrait pas omettre de mentionner l'influence indirecte du «modèle de la noce urbaine », artificiellement conçu et imposé à l'époque soviétique, mais qui a pourtant bien pénétré les pratiques contemporaines des rituels familiaux. Il ne faudrait pas non plus oublier de noter l'augmentation, aussi bien relative qu'en valeur absolue, des mariages ethniquement mixtes dans l'environnement polyethnique de la ville.

67 Malgré une tendance dominante à la désacralisation de la société, le rite nuptial préchrétien s'est fondu avec la pratique du mariage civil. Vus ainsi, les rites nuptiaux et familiaux sont un lien entre la société russe en cours de mondialisation et la culture ethnique des Maris. Ils peuvent permettre de préserver et de transmettre la culture traditionnelle marie et servent ainsi, dans une certaine mesure, d'indicateurs de son niveau d'adaptation au monde contemporain.

\section{BIBLIOGRAPHIE}

ВАВENKO Vasilij et alii 1990 = БАБЕНКО ВАСИЛИЙ ЯКОВЛЕВИЧ И ДР., СЕМЕЙНЫЕ ПРАЗДНИКИ И ОБРЯДЫ БАШКИРСКОЙ АССР (Fêtes et rituels familiaux en RSSA de Bachkirie), УФА, 1990, 10-11

JAMURZINA Ljudmila 2011 = ЯМУРЗИНА ЛЮДМИЛА, ОБРЯДЫ СЕМЕЙНОГО ЦИКЛА МАРИ В КОНТЕКСТЕ ТЕОРИИ ОБРЯДОВ ПЕРЕХОДА (НА ПРИМЕРЕ ВОСТОЧНЫХ МАРИ) (Rituels du cycle familial dans le contexte de la théorie des rites de passage (sur l'exemple des Maris orientaux), дИС....Д-РА ФИЛОСОФИИ ПО СПЕЦИАЛЬНОСТИ ЭТНОЛОГИЯ, ТАРТУ: ТАРТУРСКИЙ УНИВЕРСИТЕТ.

SEPEEV Gennadij 1967 = СЕПЕЕВ ГЕННАДИЙ АНДРЕЕВИЧ, « К ВОПРОСУ О ФОРМИРОВАНИИ ЭТНОГРАФИЧЕСКИХ ОСОБЕННОСТЕЙ ВОСТОЧНЫХ МАРИЙЦЕВ » (Sur la question de la formation des spécificités ethnographiques des Maris orientaux), ПРОИСХОЖДЕНИЕ МАРИЙСКОГО НАРОДА (La provenance du peuple mari). МАТЕРИАЛЫ НАУЧНОЙ СЕССИИ, ПРОВЕДЕННОЙ МАРНИИЯЛИ (23-25 ДЕКАБРЯ 1965 Г.), ЙОШКАР-ОЛА, МАРНИИЯЛИ, 1967. 
INDEX

nomsmotscles Maris, Maris des collines, Maris des plaines, Maris orientaux, Russes

Thèmes : anthropologie, ethnologie

Index géographique : Agryz, Bachkortostan (République), Bakaly, Baltačevo, Birsk, Bol’šoj

Ljaždur, Bol'šoj Serdež, Čibyž, Ekaterinburg, Joškar-Ola, Kaltasy, Kazan, Kokšaga,

Koz'modem'jansk, Kukmor, Mari-El (République), Marij-Turek, Mari-Kupta, Medvedevo, Mišino,

Morko, Moscou, Narat-čukur, Novyj Tor"jal, Nurma, Oršanka, Paran'ga, Počinok-Kučuk, Saint-

Pétersbourg, Sernur, Sovetskij, Tatarstan (République), Ufa, Volžsk, Zvenigovo

Mots-clés : adaptation, noce, rituels

Index chronologique : XXIe siècle

motsclesru АДАПТАЦИЯ, ОБРЯДЫ, СВАДЬБА

Keywords : Adaptation, Weddings, Rituals

motscleset kohanemine, pulm, rituaal 